\title{
Some Results on Global Stability of a Predator-Prey System*
}

\author{
Kuo-Shung Cheng, Sze-Bi Hsu, and Song-Sun Lin \\ Department of Applied Mathematics, National Chiao Tung University, Hsin-Chu, Taiwan 300, \\ Republic of China
}

\begin{abstract}
In this paper we derive some results to ensure the global stability of a predator-prey system. The results cover most of the models which have been proposed in the ecological literature for predator-prey systems. The first result is very geometric and it is very easy to check from the graph of prey and predator isoclines. The second one is purely algebraic, however, it covers the defects of the first one especially in dealing with Holling's type-3 functional response in some sense. We also discuss the global stability of Kolmogorov's model. Some examples are presented in the discussion section.
\end{abstract}

Key words: Global stability - Predator-prey system

\section{Introduction}

Global stability is a very interesting mathematical problem. Usually the biologists believe that a unique, "positive", local asymptotically stable equilibrium in an ecological model is globally stable. In this paper we rigorously prove global stability for a general class of a predator-prey system. The results cover most of the models which have been proposed in the ecological literature for predator-prey systems.

The global stability analysis for the classical Lotka-Volterra system has been given by Goh [4]. In [4], Goh constructs a Lyapunov function to prove global stability. Hsu et al. [8] employs the Dulac criterion (Bendixson's negative criterion) to prove global stability for a specific predator-prey model. In a subsequent paper, Hsu [7] presents two criteria for global stability of a general predator-prey system which was discussed by many authors ([2], [3], [9], [10], [13], for example). The first criterion was established by constructing a Lyapunov function while the second one was proved by using the Dulac criterion. Unfortunately, we found that the proof of the second criterion is not rigorously correct. We note that either constructing Lyapunov function, or using the Dulac criterion is not an easy job.

In Section 2 we state the mathematical model of a predator-prey system and present our first main result Theorem 1. The criterion in Theorem 1 is graphic and geometric. In [2], Freedman developed a graphic technique to ensure the local

* Works partially supported by the National Science Council of the Republic of China 
stability of the "positive" equilibrium. In Theorem 1 our graphic method establishes the global stability of the "positive" equilibrium. The technique used in Theorem 1 is a combination of various techniques, such as trajectory comparison between two similar systems, construction of Lyapunov-like functions and double integral evaluation by the "mirror image" method. Although the method looks complicated, it is intuitively straightforward.

In Section 3 we present our second result. The criterion is purely algebraic, however, it covers the defects of Theorem 1 in dealing with Holling's type- 3 functional response [11], [15] in some sense. The method used in Theorem 2 is an application of Floquet theory and the Poincaré-Bendixson Theorem [5]. In Section 4 , we also establish a criterion for global stability for a Kolmogorov model and discuss a non-Kolmogorov, self-regulating, predator-prey system.

In Section 5 we present two examples to show the applicability of our theorems.

\section{The Model and Global Stability}

We consider the following basic model for the predator-prey system

$$
\begin{aligned}
\frac{d x}{d t} & =x g(x)-y p(x), \\
\frac{d y}{d t} & =y[c p(x)-D], \\
x(0) & >0, \quad y(0)>0,
\end{aligned}
$$

where $x$ represents the prey population (or density), $y$ represents the predator population (or density), $g(x)$ is the specific growth rate which governs the growth of the prey in the absence of predators, $p(x)$ is the predator response function which has been much discussed in the literature and $D$ is the death rate of the predator.

The general assumptions on $g(x)$ and $p(x)$ are:

(1) $g \in C([0, \infty), R) \cap C^{1}((0, \infty), R), g(0)>0$ and there exists $K>0$ such that $g(K)=0$ and $(x-K) g(x)<0$ for $x \neq K$.

(2) $p \in C([0, \infty), R) \cap C^{1}((0, \infty), R), p(0)=0$ and $p^{\prime}(x)>0$ for all $x \geqslant 0$.

Some of the specific forms of $g(x)$ and $p(x)$ frequently used are (see [2], [9], [10], [14]):

$$
\begin{aligned}
& g(x): \quad r\left(1-\frac{x}{K}\right) ; \quad \frac{r(K-x)}{K+\varepsilon x} ; \quad r\left[1-\left(\frac{x}{K}\right)^{c}\right], \quad 1>c>0, \\
& p(x): \quad \frac{m x^{n}}{a+x^{n}}, \quad n \geqslant 1 ; \quad m x^{c}, \quad 1 \geqslant c>0 ; \quad m\left(1-e^{-c x}\right), \quad m>0 .
\end{aligned}
$$

In order to obtain a sufficient criterion for global stability, we also need the following more specific assumptions:

(3) There exists $\left(x^{*}, y^{*}\right)$ such that $c p\left(x^{*}\right)-D=0$ and

$$
\begin{gathered}
x^{*} g\left(x^{*}\right)-y^{*} p\left(x^{*}\right)=0 \quad \text { with } \quad 0<x^{*}<K, \quad y^{*}>0, \\
\frac{d}{d x}\left(\frac{x g(x)}{p(x)}\right)<0 \quad \text { for all } x^{*} \leqslant x \leqslant K,
\end{gathered}
$$


(5) $\frac{\left(2 x^{*}-x\right) g\left(2 x^{*}-x\right)}{p\left(2 x^{*}-x\right)} \leqslant \frac{x g(x)}{p(x)}$ for all $x, \max \left(0,2 x^{*}-K\right) \leqslant x \leqslant x^{*}$,

(6) $\frac{D}{p(x)}-c>c-\frac{D}{p\left(2 x^{*}-x\right)} \quad$ for all $x, x^{*} \leqslant x<\min \left\{2 x^{*}, K\right\}$.

Assumption (3) means that we have a unique "positive" equilibrium point. Assumption (4) means that the prey isocline has negative slope in the region $x^{*} \leqslant x \leqslant K$. As we shall see, this condition ensures that $\left(x^{*}, y^{*}\right)$ is locally stable. The geometric meaning of assumption (5) is that, if we take the "mirror image" of the prey isocline in the region $x^{*} \leqslant x \leqslant K$ with respect to $x=x^{*}$, then the prey isocline in the region $0 \leqslant x \leqslant x^{*}$ is above the mirror image of the prey isocline in the region $x^{*} \leqslant x \leqslant K$. This condition is very easy to check graphically. The last assumption (6) can be replaced by a more restrictive condition, that is, $1 / p(x)$ is a strictly convex function.

We note that $p^{\prime \prime}(x)<0$ implies $(1 / p(x))^{\prime \prime}>0$ and type-2 functional response [15] always satisfies the condition $(1 / p(x))^{\prime \prime}>0$. The reader can easily check that for all $p(x)$ in $(2.5)$, the condition $(1 / p(x))^{\prime \prime}>0$ is satisfied. The reader can also check that for most of the combinations between (2.2) and (2.3), condition (5) is also satisfied for wide range of $x^{*}$.

Now we have Theorem 1 .

Theorem 1. Under the assumptions (1) - (6), $\left(x^{*}, y^{*}\right)$ is globally stable for system (2.1) in the interior of the first quadrant.

To prove Theorem 1, we introduce an auxiliary system

$$
\begin{gathered}
\frac{d x}{d t}=x \bar{g}(x)-y p(x), \\
\frac{d y}{d t}=y[c p(x)-D], \\
x(0)>0, \quad y(0)>0,
\end{gathered}
$$

where the constants $c$ and $D$ and the function $p(x)$ are the same as in system (2.1) and $\bar{g}(x)$ is related to $g(x)$ as

$$
\bar{g}(x)= \begin{cases}g(x), & \text { if } x^{*} \leqslant x, \\ \frac{\left(2 x^{*}-x\right) g\left(2 x^{*}-x\right)}{p\left(2 x^{*}-x\right)} \frac{p(x)}{x} & \text { if } \max \left(0,2 x^{*}-K\right) \leqslant x \leqslant x^{*} .\end{cases}
$$

It is easy to see that we define $\bar{g}(x)$ in such a way that the prey isocline $(d x / d t=0)$ of system (2.4) is symmetric with respect to the line $x=x^{*}$.

Now we have the following lemma which follows directly from the proof in [1].

Lemma 1. Solutions of systems (2.1) and (2.4) are positive and bounded.

Lemma 2. For system (2.4) with $(1)-(6)$, if a trajectory $\gamma$ starts at $p_{0}=\left(x^{*}, y_{0}\right)$ with $y_{0}>y^{*}$ and $T>0$ is the least time such that $x(T)=x^{*}$ and $y(T)=y_{T}>y^{*}$, then $y_{0}>y_{T}$. 
Corollary. There is no periodic solution in the first quadrant for the system (2.4).

Proof. We shall prove the lemma by contradiction.

Let $\gamma$ intersect $x=x^{*}$ at $p_{1}=\left(x^{*}, y_{1}\right)$ with $y_{1}<y^{*}$ for the first time at $t=t_{1}$. Divide a path of $\gamma$ into four parts $\gamma_{1}, \gamma_{2}, \gamma_{3}$, and $\gamma_{4}$ (see Fig. la or 1b). The region $\Omega_{1}$ is bounded by $x=x^{*}, \gamma_{1}$ and $\gamma_{2}$, and $\Omega_{2}$ is bounded by $x=x^{*}, \gamma_{3}$ and $\gamma_{4}$, and $\Omega=\Omega_{1} \cup \Omega_{2}$. Let $\gamma_{i}^{\prime}=\left\{\left(x^{\prime}, y\right): x^{\prime}=2 x^{*}-x\right.$ and $\left.(x, y) \in \gamma_{i}\right\}$ be the mirror image of $\gamma_{i}$ with respect to $x=x^{*}, i=1,2$. In fact, $\gamma_{1}^{\prime}$ and $\gamma_{2}^{\prime}$ are the trajectories of

$$
\begin{gathered}
\frac{d x}{d t}=-\left[\left(2 x^{*}-x\right) \bar{g}\left(2 x^{*}-x\right)-z p\left(2 x^{*}-x\right)\right], \\
\frac{d z}{d t}=z\left[c p\left(2 x^{*}-x\right)-D\right], \quad 0 \leqslant t \leqslant t_{1}, \\
x(0)=x^{*}, \quad z(0)=y_{0} .
\end{gathered}
$$

Suppose $y_{T} \geqslant y_{0}$, we shall prove that the region $\Omega_{1}^{\prime}$ bounded by $x=x^{*}, \gamma_{1}^{\prime}$ and $\gamma_{2}^{\prime}$ is properly contained in the region $\Omega_{2}$. In fact, $\gamma_{3}$ and $\gamma_{1}^{\prime}$ are given by

$$
\frac{d y}{d x}=-\frac{y[c p(x)-D]}{p(x)\left[y-\frac{x \bar{g}(x)}{p(x)}\right]}, \quad y\left(x^{*}\right)=y_{T}
$$

and

$$
\frac{d z}{d x}=-\frac{z\left[D-c p\left(2 x^{*}-x\right)\right]}{p\left(2 x^{*}-x\right)\left[z-\frac{\left(2 x^{*}-x\right) \bar{g}\left(2 x^{*}-x\right)}{p\left(2 x^{*}-x\right)}\right]}, \quad z\left(x^{*}\right)=y_{0},
$$

respectively in the region $x^{*} \leqslant x$ and $x \bar{g}(x) / p(x) \leqslant y$.

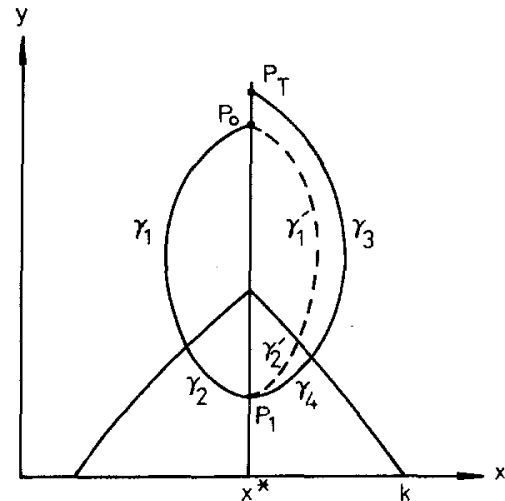

$\mathrm{Fig.1C}$

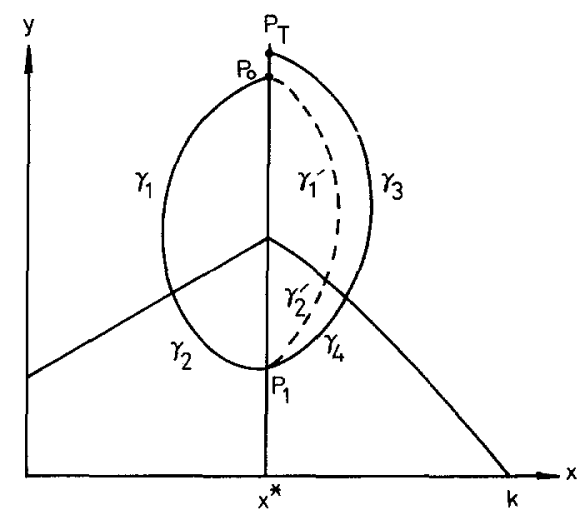

Fig.1b

Fig. 1. Phase plane in the proof of Lemma 2 
Since

$$
\frac{x \ddot{g}(x)}{p(x)}=\frac{\left(2 x^{*}-x\right) \bar{g}\left(2 x^{*}-x\right)}{p\left(2 x^{*}-x\right)} \text { for all } 0 \leqslant x \leqslant 2 x^{*},
$$

condition (6) implies that $y(x)$ satisfies the differential inequality

$$
\frac{d y}{d x}>-\frac{y\left[D-c p\left(2 x^{*}-x\right)\right]}{p\left(2 x^{*}-x\right)\left[y-\frac{\left(2 x^{*}-x\right) \bar{g}\left(2 x^{*}-x\right)}{p\left(2 x^{*}-x\right)}\right]}
$$

for $x>x^{*}$ and $y>x \bar{g}(x) / p(x)$. And so $y\left(x^{*}\right)=y_{T} \geqslant y_{0}$ implies $y(x)>z(x)$ as long as $y(x), z(x)>x \bar{g}(x) / p(x)$ (see for example [5]). Similarly, $\gamma_{4}$ and $\gamma_{2}^{\prime}$ are given by (2.7) with $y\left(x^{*}\right)=y_{1}$ and (2.8) with $z\left(x^{*}\right)=y_{1}$ in the region $x \geqslant x^{*}$ and $y<x \bar{g}(x) / p(x)$, respectively. Then by a similar argument, condition (6) implies $z(x)>y(x)$ as long as $z(x), y(x)<x \bar{g}(x) / p(x)$. This proves that $\Omega_{1}^{\prime}$ is properly contained in $\Omega_{2}$.

To obtain a contradiction by assuming $y_{T} \geqslant y_{0}$, we consider the following function $V(x, y)$

$$
V(x, y)=\int_{x^{*}}^{x} \frac{c p(\xi)-D}{p(\xi)} d \xi+\int_{y^{*}}^{y} \frac{\eta-y^{*}}{\eta} d \eta
$$

Then

$$
\dot{V}(x(t), y(t))=(c p(x)-D)\left(\frac{x \bar{g}(x)}{p(x)}-y^{*}\right) .
$$

We have

$$
\begin{aligned}
\int_{0}^{T} \dot{V}(x(t), y(t)) d t & =\left.V(x(t), y(t))\right|_{0} ^{T}=V\left(x^{*}, y_{T}\right)-V\left(x^{*}, y_{0}\right) \\
& =\int_{y_{0}}^{y_{T}} \frac{y-y^{*}}{y} d y \geqslant 0 .
\end{aligned}
$$

On the other hand, by (2.4b) and (2.10)

$$
\begin{aligned}
\int_{0}^{T} \dot{V}(x(t), y(t)) d t= & \int_{\gamma} \frac{1}{y}\left[\frac{x \bar{g}(x)}{p(x)}-y^{*}\right] d y \\
= & \int_{\gamma} \frac{1}{y}\left[\frac{x \bar{g}(x)}{p(x)}-y^{*}\right] d y+\int_{y_{T}}^{y_{0}} \frac{1}{y}\left[\frac{x^{*} \bar{g}\left(x^{*}\right)}{p\left(x^{*}\right)}-y^{*}\right] d y \\
= & \iint_{\Omega} \frac{1}{y} \frac{d}{d x}\left(\frac{x \bar{g}(x)}{p(x)}\right) d x d y=\iint_{\Omega_{1}} \frac{1}{y} \frac{d}{d x}\left(\frac{x \bar{g}(x)}{p(x)}\right) d x d y \\
& +\iint_{\Omega_{2}} \frac{1}{y} \frac{d}{d x}\left(\frac{x \bar{g}(x)}{p(x)}\right) d x d y \\
= & \iint_{\Omega_{2}-\Omega_{1}} \frac{1}{y} \frac{d}{d x}\left(\frac{x \bar{g}(x)}{p(x)}\right) d x d y<0
\end{aligned}
$$




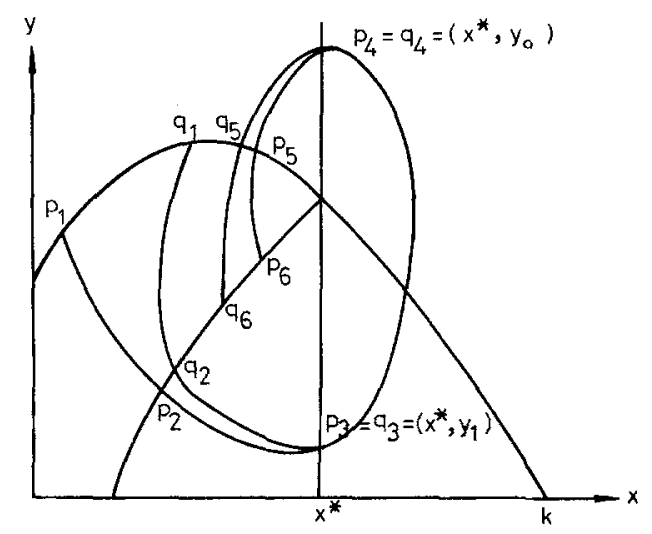

Fig. 2. Phase plane in the proof of Lemma 3

since $\Omega_{1}^{\prime}$ is properly contained in $\Omega_{2}$. This leads to a contradiction, and we have $y_{T}<y_{0}$.

Lemma 3. If we represent the systems (2.1) and (2.4) in the same phase plane (Fig. 2), let

$$
\begin{aligned}
& C_{1}=\left\{(x, y) \mid y=\frac{x g(x)}{p(x)}, 0<x<x^{*}\right\}, \\
& C_{2}=\left\{(x, y) \mid y=\frac{x \bar{g}(x)}{p(x)}, 0<x<x^{*}\right\}
\end{aligned}
$$

and consider trajectories $\Gamma_{1}$ and $\Gamma_{2}$ for system (2.1) and (2.4), respectively,

$$
\Gamma_{1}=\overbrace{p_{1} p_{2} p_{3} p_{4} p_{5} p_{6}}, \quad \Gamma_{2}=\overbrace{q_{1} q_{2} q_{3} q_{4} q_{5} q_{6}},
$$

where $p_{1}, q_{1}, p_{5}$, and $q_{5}$ belong to $C_{1}, p_{2}, q_{2}, p_{6}$, and $q_{6}$ belong to $C_{2}$, and $p_{3}=q_{3}=\left(x^{*}, y_{1}\right)$ with $y_{1}<y^{*}$ and $p_{4}=q_{4}=\left(x^{*}, y_{0}\right)$ with $y_{0}>y^{*}$, then we have $x_{p 1} \leqslant x_{q 1}<x_{q 5} \leqslant x_{p 5}$ where $x_{p i}$ and $x_{q i}$ are the $x$-coordinates of points $p_{i}$ and $q_{i}$ respectively.

Proof. By Lemma $2, x_{q 1} \leq x_{q 5}$ and so we only need to prove $x_{p 1} \leqslant x_{q 1}$ and $x_{q 5} \leqslant x_{p 5}$. Since ${\widetilde{p_{4} p_{5}}}_{1}$ and $\widetilde{q}_{4} q_{5}$ are given by

$$
\frac{d y}{d x}=\frac{y[D-c p(x)]}{p(x)\left[y-\frac{x g(x)}{p(x)}\right]}, \quad y\left(x^{*}\right)=y_{0}
$$

and

$$
\frac{d z}{d x}=\frac{z[D-c p(x)]}{p(x)\left[z-\frac{x \bar{g}(x)}{p(x)}\right]}, \quad z\left(x^{*}\right)=y_{0}
$$

in the region $x<x^{*}$ and $z, y>x g(x) / p(x)$, respectively. Then $x g(x) / p(x) \geqslant$ $x \bar{g}(x) / p(x)$ implies that $y(x)$ satisfies a differential inequality 


$$
\frac{d y}{d x} \geqslant \frac{y(x)[D-c p(x)]}{p(x)\left[y(x)-\frac{x \bar{g}(x)}{p(x)}\right]}
$$

when $x<x^{*}$ and $y>x g(x) / p(x)$. Hence, $y\left(x^{*}\right)=y_{0}=z\left(x^{*}\right)$ implies that $y(x) \leqslant z(x)$ when $x<x^{*}$ and $z(x), y(x)>x g(x) / p(x)$. Therefore, $z\left(x_{p 5}\right) \geqslant y\left(x_{p 5}\right)$ and hence $x_{q 5} \leqslant x_{p 5}$. Similarly, we can prove $x_{p 1} \leqslant x_{q 1}$.

Proof of Theorem 1. From Lemma 1, solutions of (2.1) are positive and bounded. From Lemma 3, $x_{p 1}<x_{p 5}$ for all trajectories $\Gamma_{1}$, and hence there is no periodic solution. Furthermore $\left(x^{*}, y^{*}\right)$ of $(2.1)$ is locally stable. Thus $\left(x^{*}, y^{*}\right)$ of $(2.1)$ is globally stable. This completes the proof of Theorem 1.

\section{Another Technique}

In Theorem 1 the assumption (5) is no longer true when we deal with Holling's type-3 functional response $p(x)=k x^{n} /\left(a+x^{n}\right), n \geqslant 2[11]$.

In the next theorem we present an algebraic criterion to establish global stability of $\left(x^{*}, y^{*}\right)$ for (2.1). An example concerning type-3 functional response will be discussed in the next section.

Theorem 2. Let the assumptions (1), (2), (3) in Theorem 1 hold. Assume

(i) $\left(x^{*}, y^{*}\right)$ is locally asymptotically stable, i.e., $f\left(x^{*}\right)<0$ where

$$
f(x)=\frac{d}{d x}(x g(x))-\frac{p^{\prime}(x) x g(x)}{p(x)},
$$

(ii) $\frac{f(x)-f\left(x^{*}\right)}{p(x)-p\left(x^{*}\right)}$ is $C^{1}$ and $\frac{d}{d x}\left(\frac{f(x)-f\left(x^{*}\right)}{p(x)-p\left(x^{*}\right)}\right) \leqslant 0$ for $0 \leqslant x \leqslant K$,

then $\left(x^{*}, y^{*}\right)$ is globally stable in the interior of the first quadrant.

Proof. From the Poincaré-Bendixson Theorem and Lemma 1, it suffices to show that there is no limit cycle in the interior of the first quadrant. Suppose there is a periodic orbit $\gamma=(x(t), y(t)), 0 \leqslant t \leqslant T$ with the enclosed region $\Omega$ and consider the variational matrix about the periodic orbit,

$$
\left[\begin{array}{cc}
\frac{d}{d x}(x g(x))-y p^{\prime}(x) & -p(x) \\
p^{\prime}(x) c y & c p(x)-D
\end{array}\right]_{\substack{x=x(t) \\
y=y(t)}} .
$$

Compute

$$
\Delta=\int_{0}^{T}\left[\left.\frac{d}{d x}(x g(x))\right|_{x=x(t)}-y(t) p^{\prime}(x(t))+(c p(x(t))-D] d t .\right.
$$

From the equations in $(2.1)$, it follows that

$$
\int_{0}^{T}(c p(x(t))-D) d t=0
$$


and

$$
\int_{0}^{T} y(t) p^{\prime}(x(t)) d t=\int_{0}^{T} x(t) g(x(t)) \frac{p^{\prime}(x(t))}{p(x(t))} d t .
$$

Hence we have

$$
\begin{aligned}
\Delta & =\left.\int_{0}^{T} \frac{d}{d x}(x g(x))\right|_{x=x(t)}-x(t) g(x(t)) \frac{p^{\prime}(x(t))}{p(x(t))} d t \\
& =\int_{0}^{T} f\left(x^{*}\right) d t+\int_{0}^{T} f(x(t))-f\left(x^{*}\right) d t .
\end{aligned}
$$

Furthermore

$$
\begin{aligned}
\int_{0}^{T} f(x(t))-f\left(x^{*}\right) d t & =\int_{0}^{T} \frac{f(x(t))-f\left(x^{*}\right)}{p(x(t))-p\left(x^{*}\right)} p(x(t))-p\left(x^{*}\right) d t \\
& =\frac{1}{c} \int_{0}^{T} \frac{f(x(t))-f\left(x^{*}\right)}{p(x(t))-p\left(x^{*}\right)} \frac{y^{\prime}(t)}{y(t)} d t \\
& =\frac{1}{c} \oint_{\nu} \frac{f(x)-f\left(x^{*}\right)}{p(x)-p\left(x^{*}\right)} \frac{1}{y} d y \\
& =\frac{1}{c} \int_{\Omega} \frac{1}{y} \frac{d}{d x}\left(\frac{f(x)-f\left(x^{*}\right)}{p(x)-p\left(x^{*}\right)}\right) d x d y<0 .
\end{aligned}
$$

Hence we have $\Delta<0$ and the periodic orbit $\gamma$ is orbitally asymptotically stable [5]. Since every periodic orbit is orbitally stable and then there is a unique limit cycle. From the Poincaré-Bendixson Theorem, it is impossible to have a unique stable limit cycle enclose a stable equilibrium. This is the desired contradiction. Hence there is no limit cycle and $\left(x^{*}, y^{*}\right)$ is globally stable.

Remark. Theorem 2 is also true if $f\left(x^{*}\right)=0$ and

$$
\frac{d}{d x}\left(\frac{f(x)-f\left(x^{*}\right)}{p(x)-p\left(x^{*}\right)}\right) \leqslant 0
$$

but not identically zero.

\section{Kolmogorov Model and Others}

In the next theorem, we present a criterion to establish the global stability for the Kolmogorov model. The notations and assumptions of the Kolmogorov model basically follow from [1]. We first state the hypotheses of the model. Consider

$$
\left\{\begin{array}{l}
x^{\prime}=x f(x, y), \\
y^{\prime}=y g(x, y), \quad x(0)>0, \quad y(0)>0,
\end{array}\right.
$$

where $x$ and $y$ denote the population of prey, and predator, respectively. Assume:

(p1) (a) There exists a $K>0$ such that $(x-K) f(x, 0)<0$ for all $x \geqslant 0, x \neq 0$.

(b) There exists a $\hat{y}>0$ such that $(y-\hat{y}) f(0, y)<0$ for all $y \geqslant 0, y \neq \hat{y}$. 
(c) $\partial f / \partial y<0$ in $Q^{0}$, where $Q^{0}=\{(x, y): x>0, y>0\}$.

(d) For every $(\alpha, \beta) \in Q^{0},(\partial f / \partial x)(\alpha, \beta) \alpha+(\partial f / \partial y)(\alpha, \beta) \beta<0$.

( $p 2)$ (a) There exists an $\hat{x}>0$ such that $(x-\hat{x}) g(x, 0)>0$ for all $x \geqslant 0, x \neq \hat{x}$.

(b) $\partial g / \partial y \leqslant 0$ in $Q^{0}$.

(c) For every $(\alpha, \beta) \in Q^{0},(\partial g / \partial x)(\alpha, \beta) \alpha+(\partial g / \partial y)(\alpha, \beta) \beta>0$.

(p3) $\hat{x}<K$.

Under assumptions ( $p 1),(p 2),(p 3)$, Albrecht et al. [1] show that the solution $(x(t), y(t))$ of (3.1) is positive and bounded and there exists a unique equilibrium $\left(x^{*}, y^{*}\right)$ in $Q^{0}$.

Theorem 3. Let $(p 1)-(p 3)$ hold. Assume $\left(x^{*}, y^{*}\right)$ is locally stable and

$$
x \frac{\partial f}{\partial x}(x, y)+y \frac{\partial g}{\partial y}(x, y)<0 \quad \text { for } \quad 0 \leqslant x \leqslant K, y>0 .
$$

Then $\left(x^{*}, y^{*}\right)$ is a global attractor.

Proof. It suffices to show that there is no periodic orbit. Using the same arguments as in the proof of Theorem 2, it suffices to show that if $(x(t), y(t)), 0 \leqslant t \leqslant T$ is a periodic orbit,

$$
\Delta=\int_{0}^{T}\left(\frac{\partial}{\partial x}(x f(x, y))+\left.\frac{\partial}{\partial y}(y g(x, y))\right|_{\substack{x=x(t) \\ y=y(t)}}, \quad d t<0 .\right.
$$

Since

$$
\int_{0}^{T} f(x(t), y(t)) d t=\int_{0}^{T} g(x(t), y(t)) d t=0,
$$

it follows that

$$
\Delta=\int_{0}^{T}\left(x \frac{\partial f}{\partial x}(x, y)+y \frac{\partial g}{\partial y}(x, y)\right)_{\substack{x=x(t) \\ y=y(t)}}, \quad d t<0 .
$$

Hence we completed the proof.

We note that the assumption (4.2) is consistent with the local stability of $\left(x^{*}, y^{*}\right)$ in the predator-prey system. Theorem 3 at least says that in self-regulating predator-prey system, i.e., $\partial g / \partial y<0, \partial f / \partial x<0$ in $Q^{0},\left(x^{*}, y^{*}\right)$ is a global attractor. This conclusion is similar to that in [6]. In [6] A. Hastings deals with a selfregulating, predator-prey system by the Bendixson criterion. However, we can drop the additional assumptions needed in Theorem 2 of [6] by directly introducing a Lyapunov function.

Theorem 4. Let $\partial f / \partial x<0, \partial g / \partial y<0, \partial f / \partial y<0, \partial g / \partial x>0$, in $Q^{0}$ and $\left(x^{*}, y^{*}\right)$ is the unique "positive" equilibrium in $Q^{0}$. Then $\left(x^{*}, y^{*}\right)$ is a global attractor of (4.1).

Proof. Let $F(x)=g\left(x, y^{*}\right), G(y)=-f\left(x^{*}, y\right)$ then $F(x), G(y)$ are strictly increasing functions of $x$ and $y$ respectively. Let 


$$
V=\int_{x^{*}}^{x} \frac{F(u)}{u} d u+\int_{y^{*}}^{y} \frac{G(v)}{v} d v
$$

Hence

$$
\begin{aligned}
\frac{d V}{d t}= & f(x, y) F(x)+g(x, y) G(y) \\
= & F(x)\left(f(x, y)-f\left(x^{*}, y\right)\right)+G(y)\left[g(x, y)-g\left(x, y^{*}\right)\right] \\
& +F(x) f\left(x^{*}, y\right)+G(y) g\left(x, y^{*}\right) \\
= & F(x)\left(x-x^{*}\right) \frac{\partial f}{\partial x}(\tilde{x}, y)+G(y)\left(y-y^{*}\right) \frac{\partial g}{\partial y}(x, \tilde{y}) \leqslant 0,
\end{aligned}
$$

where $\tilde{x}$ is between $x$ and $x^{*}$ and $\tilde{y}$ is between $y$ and $y^{*}$. By LaSalle's Theorem [5], $\left(x^{*}, y^{*}\right)$ is a global attractor.

\section{Discussion and Examples}

The biological assumption for predator-prey system (2.1) is that the predators do not actually interfere with each other directly. Theorem 1 can be interpreted graphically: (i) the prey-isocline is decreasing for $x^{*} \leqslant x \leqslant K$, (ii) the curve obtained by reflecting the curve in (i) symmetrically with respect to $x=x^{*}$ is always below the prey-isocline.

Our first example is a typical example from the ecological literature. The functional response is $p(x)=m x^{\beta}, 0<\beta \leqslant 1$, for a reference see Rosenzweig [14].

\section{Example 1.}

$$
\begin{aligned}
& \frac{d x}{d t}=\gamma x\left(1-\frac{x}{K}\right)-m x^{\beta} y, \\
& \frac{d y}{d t}=y\left[c m x^{\beta}-D\right] .
\end{aligned}
$$

The prey isocline is

$$
y=\frac{\gamma}{m} x^{1-\beta}\left(1-\frac{x}{K}\right) .
$$

If $\beta \geqslant 1$, then from Theorem $1\left(x^{*}, y^{*}\right)$ is globally stable for any $0<x^{*}<K$.

If $0<\beta<1$, we prove that $\left(x^{*}, y^{*}\right)$ is globally stable by applying Theorem 2 as follows:

$$
\begin{aligned}
p(x)-p\left(x^{*}\right) & =m\left[x^{\beta}-\left(x^{*}\right)^{\beta}\right] \\
f(x) & =\gamma\left(1-\frac{2 x}{K}\right)-\gamma x\left(1-\frac{x}{K}\right) \frac{m c x^{\beta-1}}{m x^{\beta}}, \\
& =\gamma\left[\left(1-\frac{2 x}{K}\right)-\beta\left(1-\frac{x}{K}\right)\right],
\end{aligned}
$$




$$
\begin{aligned}
& f(x)-f\left(x^{*}\right)=\frac{\gamma}{K}(\beta-2)\left(x-x^{*}\right), \\
& \frac{f(x)-f\left(x^{*}\right)}{p(x)-p\left(x^{*}\right)}=\frac{\gamma}{m K}(\beta-2) \frac{x-x^{*}}{x^{\beta}-\left(x^{*}\right)^{\beta}} .
\end{aligned}
$$

For $0<\beta \leqslant 1$, it suffices to show that

$$
\begin{gathered}
\frac{d}{d x} \frac{x-x^{*}}{x^{\beta}-\left(x^{*}\right)^{\beta}} \geqslant 0 . \\
\frac{d}{d x} \frac{x-x^{*}}{x^{\beta}-\left(x^{*}\right)^{\beta}}=\frac{1}{\left(x^{\beta}-\left(x^{*}\right)^{\beta}\right)^{2}}\left[(1-\beta) x^{\beta}+\beta x^{*} x^{\beta-1}-\left(x^{*}\right)^{\beta}\right]
\end{gathered}
$$

claim :

$$
h(x)=(1-\beta) x^{\beta}+c x^{*} x^{\beta-1}-\left(x^{*}\right)^{\beta} \geqslant 0 \quad \text { for } \quad 0 \leqslant x \leqslant K .
$$

We have $h\left(x^{*}\right)=0, h^{\prime}(x)=x^{\beta-2} \beta(1-\beta)\left[x-x^{*}\right]$, thus $h^{\prime}(x) \geqslant 0$ as $x \geqslant x^{*}$ and $h^{\prime}(x)<0$ as $0<x<x^{*}$.

Q.E.D.

As we pointed out in Section 3, Theorem 1 no longer holds for Holling's type-3 functional response. The following example is suggested by [11]. It also generalizes the model in [8].

\section{Example 2.}

$$
\left\{\begin{array}{l}
\frac{d x}{d t}=\gamma x\left(1-\frac{x}{k}\right)-\frac{m x^{n} y}{a+x^{n}} \\
\frac{d y}{d t}=\left[c \frac{m x^{n}}{a+x^{n}}-D\right] y
\end{array}\right.
$$

$n$ is a positive integer.

By scaling the population $y$, we may assume $c=1$. From Theorem 2

$$
\begin{aligned}
p(x)-p\left(x^{*}\right) & =\frac{m a}{a+\left(x^{*}\right)^{n}} \frac{x^{n}-\left(x^{*}\right)^{n}}{a+x^{n}} \\
f(x) & =\frac{d}{d x}(x g(x))-x g(x) \frac{p^{\prime}(x)}{p(x)}=\gamma\left[\left(1-\frac{2 x}{k}\right)-\left(1-\frac{x}{k}\right) \frac{n a}{a+x^{n}}\right] .
\end{aligned}
$$

With some calculations one can show

$$
\begin{aligned}
f(x)-f\left(x^{*}\right)= & \gamma\left[-\frac{2}{k}\left(x-x^{*}\right)-n a\left\{\begin{array}{l}
1-\frac{x}{k} \\
\frac{1}{a+x^{n}}-\frac{x^{*}}{a+\left(x^{*}\right)^{n}}
\end{array}\right\}\right] \\
= & -\frac{\gamma}{k} \frac{1}{\left(a+x^{n}\right)\left(a+\left(x^{*}\right)^{n}\right)}\left[\left(2 x^{n}+a(2-n)\right)\right. \\
& \left.\times\left(a+\left(x^{*}\right)^{n}\right)\left(x-x^{*}\right)+n a\left(x^{*}-k\right)\left(x^{n}-\left(x^{*}\right)^{n}\right)\right] .
\end{aligned}
$$

Thus 


$$
\frac{d}{d x}\left(\frac{f(x)-f\left(x^{*}\right)}{p(x)-p\left(x^{*}\right)}\right)=-\frac{\gamma}{k} \frac{1}{m a}\left(a+\left(x^{*}\right)^{n}\right) \frac{d}{d x}\left[\frac{\left(2 x^{n}+a(2-n)\right)\left(x-x^{*}\right)}{x^{n}-\left(x^{*}\right)^{n}}\right] .
$$

It suffices to show

$$
\Delta=\frac{d}{d x}\left[\frac{\left(2 x^{n}+a(2-n)\right)\left(x-x^{*}\right)}{x^{n}-\left(x^{*}\right)^{n}}\right] \geqslant 0 .
$$

For $n=1$ it is trivial. For $n \geqslant 2$,

$$
x^{n}-\left(x^{*}\right)^{n}=\sum_{\substack{j+k=n-1 \\ 0 \leqslant j, k \leqslant n-1}} x^{j}\left(x^{*}\right)^{k}\left(x-x^{*}\right) .
$$

Then

$$
\begin{aligned}
\Delta= & \frac{1}{\left(\sum x^{j}\left(x^{*}\right)^{k}\right)^{2}}\left[2 n x ^ { n - 1 } \left(\left(x^{*}\right)^{n-1}+\sum_{\substack{j+k=n-2 \\
0 \leqslant j, k \leqslant n-2}} x^{j+1}\left(x^{*}\right)^{k}\right.\right. \\
& \left.-\left(2 x^{n}+a(2-n)\right) \sum_{\substack{j+k=n-2 \\
0 \leqslant j, k \leqslant n-2}}(j+1) x^{j}\left(x^{*}\right)^{k}\right] \\
> & \frac{1}{\left(\sum x^{j}\left(x^{*}\right)^{k}\right)^{2}}\left[-a(2-n) \sum(j+1) x^{j}\left(x^{*}\right)^{k}\right. \\
& \left.+\sum_{\substack{j+k=n-2 \\
0 \leqslant j, k \leqslant n-2}}(2 n-2(j+1)) x^{n+j}\left(x^{*}\right)^{k}\right]>0 .
\end{aligned}
$$

Hence by Theorem $2,\left(x^{*}, y^{*}\right)$ is a global attractor.

\section{References}

1. Albrecht, F., Gatzke, H., Haddad, A., Wax, N.: The dynamics of two interacting populations. J. Math. Anal. Appl. 46, 658-670 (1974)

2. Freedman, H. I.: Graphical stability, enrichment, and pest control by a natural enemy. Math. Biosci. 31, 207-225 (1976)

3. Gaus, G. F., Smaragdova, N. P., Witt, A. A.: Further studies of interaction between predators and prey. J. Anim. Ecol. 5, 1-18 (1936)

4. Goh, B. S.: Global stability in many species systems. Amer. Natur. 111 (977), 135-143 (1977)

5. Hale, J. K.: Ordinary differential equations. New York: Wiley-Interscience 1969

6. Hastings, A.: Global stability of two species system. J. Math. Biol. 5, 399-403 (1978)

7. Hsu, S. B.: On global stability of a predator-prey system. Math. Biosci. 39, 1-10 (1978)

8. Hsu, S. B., Hubbel, S. P., Waltman, P.: Competing predators. SIAM J. Applied Math. 35, 617-625 (1978)

9. May, R. M.: Stability and complexity in model ecosystems. Princeton, U.P., Princeton, N.J., 1974

10. Oaten, A., Murdoch, W. W.: Functional response and stability in predator-prey system. Amer. Natur. 109, $289-298$ (1975)

11. Real, L. A.: The kinetics of functional response. Amer. Natur. 111 (1978), 289-300 (1977)

12. Rosenzweig, M. L.: Why the prey curve has a hump. Amer. Natur. 103, $81-87$ (1969)

13. Rosenzweig, M. L., MacArthur, R. H.: Graphical representation and stability conditions of predator-prey interaction. Amer. Natur. 97, 209-223 (1963)

14. Rosenzweig, M. L.: Paradox of enrichment: Destabilization of exploitation ecosystem in ecological time. Science 171, 385-387 (1971)

15. Maynard Smith, J.: Models in ecology. Cambridge: University Press 1974

Received April 23/Revised November 24, 1980 